\title{
A class of hyperbolic-parabolic coupled systems applied to image restoration
}

Junling Sun ${ }^{1,2}$, Jie Yang ${ }^{1 *}$ and Lei Sun ${ }^{2}$

\section{"Correspondence:}

Yangjie196002@163.com

'School of Information Engineering,

Wuhan University of Technology,

Wuhan, 430070, People's Republic of China

Full list of author information is

available at the end of the article

\begin{abstract}
This paper considers a coupled system with a new kind of hyperbolic-parabolic partial differential equations based on image restoration. We show that this system has global dissipative solutions under Dirichlet boundary conditions and initial condition. Meanwhile, an experimental approach is given to show the efficiency of this kind of model.
\end{abstract}

MSC: 35K57; 35B32; 92D25

Keywords: image restoration; parabolic equation; hyperbolic equation; nonlinear diffusion; dissipative solution

\section{Introduction}

The present paper considers the hyperbolic-parabolic system

$$
\begin{aligned}
& \frac{\partial u}{\partial t}-\operatorname{div}(g(v) \nabla u)=0, \\
& \frac{\partial^{2} v}{\partial t^{2}}+\frac{\partial v}{\partial t}-\lambda \operatorname{div}(\nabla v)-(1-\lambda)(|\nabla u|-v)=0,
\end{aligned}
$$

subject to the initial condition and Dirichlet boundary conditions

$$
\begin{aligned}
& u(x, 0)=u_{0}(x), \quad v(x, 0)=v_{0}(x), \quad \frac{\partial v}{\partial t}(x, 0)=0, \quad x \in \Omega, \\
& \left.\frac{\partial u}{\partial n}\right|_{\partial \Omega}=0,\left.\quad \frac{\partial v}{\partial n}\right|_{\partial \Omega}=0, \quad 0<t<T,
\end{aligned}
$$

where $\Omega$ is a bounded domain of $\mathbf{R}^{n}$ with appropriately smooth boundary, $n$ is the unit outer normal to $\Omega, T>0$, and $\lambda>0$. The nonlinear term $g(s)$ obeys

$$
g(s)=\frac{1}{1+\left(\frac{s}{K}\right)^{2}} \quad \text { or } \quad g(s)=|s|^{-1}
$$

with $K>0$.

Parabolic partial differential equations based image restoration is a powerful method to deal with the trade-off between noise removal and edge preservation. This method is now

(c) 2016 Sun et al. This article is distributed under the terms of the Creative Commons Attribution 4.0 International License (http://creativecommons.org/licenses/by/4.0/), which permits unrestricted use, distribution, and reproduction in any medium, provided you give appropriate credit to the original author(s) and the source, provide a link to the Creative Commons license, and indicate if changes were made. 
a well-researched area within the image processing community. The most powerful model is the parabolic model with variable coefficient

$$
\frac{\partial u}{\partial t}-\operatorname{div}(c(x, y) \nabla u)=0,
$$

where the degree of denoising and preservation of singularities can be determined by changing $c(x, y)$. There are other types of parabolic equations, such as anisotropic diffusion models [1, 2], complex diffusion models [3], fourth order equation models [4, 5], and total variation models [6-8]. In Perona-Malik [2] the denoising capabilities of the linear diffusion can be better, let $c(x, y)=g(|\nabla u|)$ and initial data $u(0)=u_{0}$. Here the diffusion smooth function $g:[0, \infty) \longrightarrow[0, \infty)$ is important in controlling the smoothing and even enhancement of edges. They mainly considered the following two diffusion functions:

$$
g(s)=\frac{1}{1+\left(\frac{s}{K}\right)^{2}} \quad \text { or } \quad g(s)=e^{-\left(\frac{s}{K}\right)^{2}} \quad \text { with } K>0 .
$$

Catte et al. [9] first introduced a new modification and proved its well-posedness to make the gradient computation robust outliers and provide a smooth edge map for the diffusion operator. This makes the Perona-Malik type PDE better. We have

$$
\frac{\partial u}{\partial t}-\operatorname{div}\left(g\left(\left|G_{\sigma} \star \nabla u\right|\right) \nabla u\right)=0,
$$

where

$$
G_{\sigma}(x)=(2 \pi \sigma)^{-1} e^{-\frac{|x|^{2}}{2 \sigma}}
$$

is the Gaussian kernel and the operation means convolution.

Ratner and Zeevi [10] introduced a new telegraph-diffusion model

$$
\frac{\partial^{2} u}{\partial t^{2}}+\lambda \frac{\partial u}{\partial t}-\operatorname{div}(c(x, y) \nabla u)=0
$$

to describe the contraction and fluctuation of the image create denoising and edge preserving effect. This model is based on viewing the image as an elastic sheet.

A coupled parabolic equations was introduced to create better edge maps (see [11, 12]), which has the following form:

$$
\begin{aligned}
& \frac{\partial u}{\partial t}-\operatorname{div}(g(v) \nabla u)=0 \\
& \frac{\partial v}{\partial t}-\Delta v=0
\end{aligned}
$$

In order to localize denoising effects in the diffusion process based scheme, Nitzberg and Shiota [13] introduced the following relaxation model:

$$
\frac{\partial u}{\partial t}-\operatorname{div}(g(v) \nabla u)=0
$$




$$
\frac{\partial v}{\partial t}-\lambda G_{\sigma} \star|\nabla u|^{2}-\lambda v=0
$$

where $\lambda>0$ is the relaxation parameter.

Recently, Surya Prasath and Vorotnikov [14] improved the above model and provided some new modifications. One of them has caught our attention, as follows:

$$
\begin{aligned}
& \frac{\partial u}{\partial t}-\operatorname{div}(g(v) \nabla u)=0, \\
& \frac{\partial v}{\partial t}-\lambda \operatorname{div}(\nabla v)-(1-\lambda)(|\nabla u|-v)=0,
\end{aligned}
$$

where $g(s)=\frac{1}{1+\left(\frac{s}{K}\right)^{2}}$ (Perona-Malik type diffusion function) or $g(s)=|s|^{-1}$ (total variation diffusion function). $0 \leq \lambda \leq 1$ is a balancing parameter. The first equation is usually used in Perona-Malik type PDEs. In their discussion, the above model is in favor of preservation of edges. However, when the noise is very large, the preservation of edges will be unstable, which is similar to that of the Perona-Malik model.

To the best of our knowledge, this is the first work which considers a coupled hyperbolicparabolic system as a method based on viewing the image as an elastic sheet to improve the quality of the detected edges. As is well known, most of these schemes use the absolute value of the gradient image as a guiding road map in the diffusion process to restore noisy images. One can see $[9,15-20]$ for more details.

This paper is organized as follows. In Section 2 we study the existence and uniqueness of solutions of the problem (1.1)-(1.5). In Section 3 we give some numerical experiments.

\section{Existence of dissipative solutions and weak solutions}

This section is devoted to establishing the existence, uniqueness, and regularity of dissipative solutions to the problem (1.1)-(1.5). Let $L^{p}(\Omega), W_{p}^{m}(\Omega)$, and $H^{m}(\Omega)$ be the Lebesgue and Sobolev spaces. For convenience, we use the function space symbol and omit $\Omega$. The Euclidean norm in finite-dimensional spaces and $L^{2}(\Omega)$ are denoted by $|\cdot|$ and $L^{2}(\Omega)$, respectively. The corresponding scalar products is denoted by a $\cdot$ and parentheses, $(\cdot, \cdot)$. Let $H_{0}^{1}(\Omega)$ be the closure of the smooth set, which is compactly supported in $\Omega$. By means of the Friedrichs inequality, $\|\cdot\|_{1}$ corresponding to the scalar product $(u, v)_{1}=(\nabla u, \nabla v)$ is a norm in $H_{0}^{1}$. Then we collect some standard Sobolev inequalities.

The usual Sobolev inequality is

$$
\|u\|_{L_{\infty}} \leq C(\Omega)\|u\|_{2}, \quad \forall u \in V_{2} .
$$

The Ladyzhenskaya inequality is

$$
\left\|u^{2}\right\| \leq \sqrt{2}\|u\|\|\nabla u\|, \quad \forall u \in H_{0}^{1}
$$

Let $V_{r}$ be the closure of $V_{2}$ in $W_{r}^{1}$ with $1<r<2$, where $V_{2}=H_{0}^{1}(\Omega) \cap H^{2}(\Omega)$ is a Hilbert space with the scalar product

$$
(u, v)_{2}=(u, v)_{1}+\sum_{|\alpha|=2}\left(D^{\alpha} u, D^{\alpha} v\right) .
$$


We will consider our problem in the following space:

$$
W_{1}=W_{1}(\Omega, T)=\left\{u \in L_{2}\left(0, T ; V_{2}\right), u^{\prime} \in L_{2}\left(0, T ; V_{2}^{*}\right)\right\}
$$

with the norm

$$
\|u\|_{W_{1}}=\|u\|_{L_{2}\left(0, T ; V_{2}\right)}+\left\|u^{\prime}\right\|_{L_{2}\left(0, T ; V_{2}^{*}\right)}
$$

and

$$
W_{2}=W_{2}(\Omega, T)=\left\{u \in L_{2}\left(0, T ; H_{0}^{1}\right), u^{\prime} \in L_{2}\left(0, T ; H^{-1}\right)\right\}
$$

with the norm

$$
\|u\|_{W_{2}}=\|u\|_{L_{2}\left(0, T ; H_{0}^{1}\right)}+\left\|u^{\prime}\right\|_{L_{2}\left(0, T ; H^{-1}\right)}
$$

We also need the following class of pairs of functions:

$$
\begin{aligned}
\mathbb{R}= & L_{4, \mathrm{loc}}\left(0, \infty ; V_{2}\right) \cap L_{\infty}\left(0, \infty ; W_{\infty}^{1}\right) \cap W_{4, \mathrm{loc}}^{1}\left(0, \infty ; L_{2}\right) \times L_{2, \mathrm{loc}}\left(0, \infty ; V_{2}\right) \\
& \cap L_{\infty}\left(0, \infty ; L_{\infty}\right) \cap W_{2, \mathrm{loc}}^{1}\left(0, \infty ; L_{2}\right) .
\end{aligned}
$$

Define

$$
\begin{aligned}
& E_{1}(u, v, v)=-\frac{\partial u}{\partial t}-v \operatorname{div}(g(v) \nabla u), \\
& E_{2}(u, v, v)=-\frac{\partial^{2} v}{\partial t^{2}}-\frac{\partial v}{\partial t}+\lambda \operatorname{div}(\nabla v)+v(1-\lambda)(|\nabla u|-v)+(1-\delta)(\nabla v, \nabla \lambda), \\
& E_{1}(u, v)=E_{1}(u, v, 1), \\
& E_{2}(u, v)=E_{2}(u, v, 1),
\end{aligned}
$$

where $v$ is a positive constant and $\forall(u, v) \in \mathbb{R}$.

Definition 2.1 A pair of functions $(u, v) \in C_{w}\left([0, \infty) ; L_{2}\right)$ is called a dissipative solution of the problem (1.1)-(1.2), if $\forall$ test functions $(\psi, \phi) \in \mathbb{R}$, one has

$$
\begin{aligned}
& \gamma^{\|u(t)\|^{2}}\left[\|u(t)-\psi(t)\|^{2}+\|v(t)-\phi(t)\|^{2}+\left\|v^{\prime}(t)-\phi^{\prime}(t)\right\|^{2}\right] \\
& \leq \gamma^{2 t+\left\|u_{0}\right\|^{2}}\left\{\|u(0)-\psi(0)\|^{2}+\|v(0)-\phi(0)\|^{2}+\left\|v^{\prime}(0)-\phi^{\prime}(0)\right\|^{2}\right. \\
& \left.\quad+\int_{0}^{t} 2 \gamma^{-s}\left|\left(E_{1}(\psi, \phi)(s), u(s)-\psi(s)\right)+\left(E_{2}(\psi, \phi)(s), v(s)-\phi(s)\right)\right|\right\},
\end{aligned}
$$

where $v^{\prime}(t)=\frac{d v}{d t}, u_{0}, v_{0}, v_{0}^{\prime} \in L_{2}(\Omega)$, and $\gamma>1$ is a certain function of $\Omega, g, \lambda, \psi$, and $\phi$.

Definition 2.2 A pair of functions $(u, v) \in W_{1} \times W_{2}$ is called weak solutions of the problem (1.1)-(1.2), if $\forall$ test functions $(\psi, \phi) \in V_{2} \times H_{0}^{1}$,

$$
\frac{d}{d t}(u, \psi)+\epsilon(u, \psi)_{2}+v(g(v) \nabla u, \nabla \psi)=0,
$$




$$
\frac{d}{d t}\left(v^{\prime}, \phi^{\prime}\right)+\frac{d}{d t}(v, \phi)+\lambda(\nabla v, \nabla \phi)+v(\nabla v, \phi \nabla v)-v(1-\lambda)(|\nabla u|-v, \phi)=0,
$$

holding almost everywhere in $(0, T)$.

Now we state our main result.

Theorem 2.3 The problem (1.1)-(1.2) with conditions (1.3)-(1.4) admits a dissipative solution $(u, v) \in L_{\frac{4}{3}, \text { loc }}\left(0, \infty ; V_{-\epsilon+\frac{4}{3}}\right) \times H^{1}\left(0, \infty ; H_{0}^{1}\right)$ with the initial data $u_{0}, v_{0}, v_{0}^{\prime} \in L_{2}$, and $0<\epsilon<\frac{1}{3}$. Moreover, if there exists a strong solution $\left(u_{T}, v_{T}\right)$ of the problem (1.1)-(1.2), then the restriction of any dissipative solution to $(0, T)$ coincides with $\left(u_{T}, v_{T}\right)(T>0)$. Every strong solution $(u, v) \in \mathbb{R}$ is a unique dissipative solution.

To prove our main result, we introduce the following auxiliary problem:

$$
\begin{aligned}
& \frac{\partial u}{\partial t}=v \operatorname{div}(g(v) \nabla u) \\
& \frac{\partial^{2} v}{\partial t^{2}}+\frac{\partial v}{\partial t}-\lambda \operatorname{div}(\nabla v)=v(1-\lambda)(|\nabla u|-v)+(1-\delta)(\nabla v, \nabla \lambda)
\end{aligned}
$$

with the conditions

$$
\begin{aligned}
& u(x, 0)=v u_{0}(x), \quad v(x, 0)=v v_{0}(x), \quad v^{\prime}(x, 0)=v v_{0}^{\prime}(x), \quad x \in \Omega, \\
& \left.\frac{\partial u}{\partial n}\right|_{\partial \Omega}=0,\left.\quad \frac{\partial v}{\partial n}\right|_{\partial \Omega}=0, \quad 0<t<T .
\end{aligned}
$$

Lemma 2.4 Let $\left(u_{0}, v_{0}, v_{0}^{\prime}\right) \in L_{2} \times L_{2}$ and $T$ be positive constant. Then the problem (2.3)(2.4) admits a weak solution with $v=1$.

Proof We define the operators $\mathcal{A}$ and $\mathcal{B}$ from $W_{1} \times W_{2}$ to $L_{2}\left(0, T ; V_{2}^{*}\right) \times H^{1}\left(0, T ; H^{-1}\right) \times$ $L_{2} \times L_{2} \times L_{2}$ by

$$
\begin{aligned}
& (\mathcal{A}(u, v),(\psi, \phi)) \\
& \quad=\left(\frac{d}{d t}(u, \psi)+\epsilon(u, \psi)_{2}, \frac{d}{d t}\left(v^{\prime}, \phi^{\prime}\right)+\frac{d}{d t}(v, \phi)+(\lambda \nabla v, \nabla \phi),\left.u\right|_{t=0},\left.v\right|_{t=0},\left.v^{\prime}\right|_{t=0}\right), \\
& (\mathcal{B}(u, v),(\psi, \phi))=\left(-(g(v) \nabla u, \nabla \psi),-(c(x) \nabla v, \phi \nabla \lambda)+(1-\lambda)(|\nabla u|-v, \phi), u_{0}, v_{0}\right),
\end{aligned}
$$

where $(\psi, \phi) \in V_{2} \times H_{0}^{1}$ is a pair of test functions.

Then we can rewrite the problem (2.3)-(2.4) as the weak statement

$$
\mathcal{A}(u, v)=v \mathcal{B}(u, v)
$$

Note that $\mathcal{B}$ is continuous and compact, $W_{1} \subset L_{p}\left(0, T ; W_{p}^{1}\right)$ is compact for some $p>2$, and $W_{2} \subset L_{p}\left(0, T ; L_{2}^{1}\right)$ (see [21]). If

$$
\left(u_{n}, v_{n}\right) \longrightarrow\left(u_{0}, v_{0}\right), \quad \text { weakly in } W_{1} \times W_{2},
$$


then we have

$$
\left(u_{n}, v_{n}\right) \longrightarrow\left(u_{0}, v_{0}\right), \quad \text { strongly in } L_{p}\left(0, T ; W_{P}^{1}\right) \times H^{1}\left(0, T ; L_{2}\right)
$$

By Krasnoselskii's theorem [22], we have

$$
g\left(v_{n}\right) \longrightarrow g\left(v_{0}\right) \quad \text { in } L_{p}\left(0, T ; L_{q}\right), \forall q<+\infty .
$$

So

$$
g\left(v_{n}\right) \nabla u_{n} \longrightarrow g\left(v_{0}\right) \nabla u_{0} \quad \text { in } L_{p}\left(0, T ; L_{2}\right) .
$$

Since the linear operator $\mathcal{A}$ is continuous and invertible (see [14]), we can rewrite (2.7) as

$$
(u, v)=v \mathcal{A}^{-1} \mathcal{B}(u, v) \quad \text { in } W_{1} \times W_{2} .
$$

Now we derive the following estimate:

$$
\begin{aligned}
\gamma^{\|u(t)\|^{2}}\left[\|u(t)-\psi(t)\|^{2}+\|v(t)-\phi(t)\|^{2}+\left\|v^{\prime}(t)-\phi^{\prime}(t)\right\|^{2}\right. \\
\left.\quad+2 \epsilon \int_{0}^{t}\|u(s)-\psi(s)\|_{2}^{2} d s+\lambda_{0} \int_{0}^{t}\|v(s)-\phi(s)\|_{1}^{2} d s\right] \\
\leq \gamma^{2 t+v\left\|u_{0}\right\|^{2}}\left\{\|v u(0)-\psi(0)\|^{2}+\|v v(0)-\phi(0)\|^{2}+\left\|v v^{\prime}(0)-\phi^{\prime}(0)\right\|^{2}\right. \\
\quad+\int_{0}^{t} 2 \gamma^{-s} \mid\left(E_{1}(\psi, \phi, v)(s), u(s)-\psi(s)\right) \\
\left.\quad+\left(E_{2}(\psi, \phi, v)(s), v(s)-\phi(s)\right)-\epsilon(\psi(s), u(s)-\psi(s))_{2} \mid d s\right\}
\end{aligned}
$$

where $\gamma>1$ is a certain function of $\Omega, g, \lambda, \mu, \psi$, and $\phi$.

To prove the above estimate, we need to carry out an energy estimate. Let $\psi(t)=u(t)$ and $\phi(t)=v(t)$ in (2.1)-(2.2), respectively, and we have

$$
\begin{aligned}
& \frac{1}{2} \frac{d}{d t}(u, u)+\epsilon(u, u)_{2}+v(g(v) \nabla u, \nabla u)=0, \\
& \frac{d}{d t}\left(v^{\prime}, v^{\prime}\right)+\frac{d}{d t}(v, v)+\lambda(\nabla v, \nabla v)+v(\nabla v, v \nabla v)-v(1-\lambda)(|\nabla u|-v, v)=0 .
\end{aligned}
$$

Summing up (2.10)-(2.11) and integrating over $(0, t)$, we get

$$
\begin{aligned}
& \frac{1}{2}\left(\|u\|^{2}+\|v\|^{2}+\left\|v^{\prime}\right\|^{2}\right)+\int_{0}^{t} v(g(v) \nabla u, \nabla u) d s \\
& \quad+\int_{0}^{t} \lambda(\nabla v, \nabla v)+v(\nabla v, v \nabla v)-v(1-\lambda)(|\nabla u|-v, v) d s \\
& \quad \leq \frac{v}{2}\left(\left\|u_{0}\right\|^{2}+\left\|v_{0}\right\|^{2}\right) .
\end{aligned}
$$


On the other hand, $\forall(\eta, \theta)$ test function in $V_{2} \times H_{0}^{1}$, we have

$$
\begin{aligned}
& \frac{d}{d t}(\psi, \eta)+v(g(\phi) \nabla \psi, \nabla \eta)+\left(E_{1}(\psi, \phi, v), \eta\right)+\epsilon(\psi, \eta)_{2}=\epsilon(\psi, \eta), \\
& \frac{d}{d t}\left(\phi^{\prime}, \theta^{\prime}\right)+\frac{d t}{d t}(\phi, \theta)+(\lambda \nabla \phi, \theta)+v(\nabla \phi, \theta \nabla \lambda) \\
& \quad-v(1-\lambda)(|\nabla \psi|-\phi, \theta)+\left(E_{2}(\psi, \phi, \lambda), \theta\right)=0 .
\end{aligned}
$$

Let $\eta=u-\psi$ and $\theta=v-\phi$. Summing up (2.13)-(2.14) and noticing (2.12), we get

$$
\begin{aligned}
& \frac{1}{2} \frac{d}{d t}\left((\eta, \eta)+(\theta, \theta)+\left(\theta^{\prime}, \theta^{\prime}\right)\right)+v(g(v) \nabla \eta, \nabla \eta)+\epsilon(\eta, \eta)_{2}+(\lambda \nabla \theta, \nabla \theta)+v((1-\lambda) \theta, \theta) \\
& =-v([g(v)-g(\phi)] \nabla \theta, \nabla \eta)+v(1-\lambda)(|\nabla u|-|\nabla \theta|, \theta)-v(\nabla \theta, \theta \nabla \lambda) \\
& \quad+\left(E_{1}(\psi, \phi, v), \eta\right)+\left(E_{2}(\psi, \phi, \lambda), \theta\right)-\epsilon(\eta, \theta)_{2}
\end{aligned}
$$

It is easy to derive that

$$
\begin{aligned}
& -v([g(v)-g(\phi)] \nabla \theta, \nabla \eta)+v(1-\lambda)(|\nabla u|-|\nabla \theta|, \theta) \\
& \quad \leq C(\psi, g) v(|v-\phi|,|\nabla \eta|) \\
& \quad \leq\|\sqrt{v g(v)} \nabla \eta\|^{2}+C(\psi, \phi, g)\|\theta\|^{2}+C(\psi, g)\left(\theta^{2}, \sqrt{v g(v) \mid}|\nabla u|\right)
\end{aligned}
$$

and

$$
-v(\nabla \theta, \theta \nabla \lambda) \leq \frac{\lambda_{0}}{4}\|\theta\|_{1}^{2}+C(\lambda)\|\theta\|^{2} .
$$

Thus, applying (2.16)-(2.17) to (2.15), we derive

$$
\begin{aligned}
\frac{1}{2} \frac{d}{d t} & \left((\eta, \eta)+(\theta, \theta)+\left(\theta^{\prime}, \theta^{\prime}\right)\right)+\epsilon(\eta, \eta)_{2}+\frac{3 \lambda_{0}}{4} \mu^{-1}\|\theta\|_{1}^{2} \\
\leq & C(\theta, \phi, \lambda, g)\left(\theta^{2}, 1+\sqrt{v g(v)}|\nabla u|\right) \\
& +\left(E_{1}(\psi, \phi, v), \eta\right)+\left(E_{2}(\psi, \phi, \lambda), \theta\right)-\epsilon(\eta, \theta)_{2} .
\end{aligned}
$$

Denote $\Phi(t)=\|1+\sqrt{v g(v)}|\nabla u|\|$. Then it follows from (2.18) that

$$
\begin{aligned}
\frac{1}{2} \frac{d}{d t}\left(\|\eta\|^{2}+\|\theta\|^{2}+\left\|\theta^{\prime}\right\|^{2}\right)+2 \epsilon\|\eta\|_{2}^{2}+\lambda_{0} \mu^{-1}\|\nabla \theta\|^{2} \\
\leq C(\theta, \phi, \lambda, g) \Phi^{2}\|\theta\|^{2} \\
\quad+2\left(E_{1}(\psi, \phi, \nu), \eta\right)+2\left(E_{2}(\psi, \phi, \lambda), \theta\right)-2 \epsilon(\eta, \theta)_{2}
\end{aligned}
$$

By (2.13), we have

$$
t|\Omega| \leq \int_{0}^{t} \Phi^{2}(s) d s \leq 2 t|\Omega|+v\left\|u_{0}\right\|^{2}-\|u(t)\|^{2} .
$$

Hence, using (2.19)-(2.20) and a Gronwall-type inequality, we obtain (2.9). 
Let $\eta=\theta=0$. It is easy to see that

$$
\begin{aligned}
& \|u\|_{L_{\infty}\left(0, T ; L_{2}\right)}+\|v\|_{L_{\infty}\left(0, T ; L_{2}\right)}+\|v\|_{L_{2}\left(0, T ; H_{0}^{1}\right)} \leq C, \\
& \|u\|_{L_{\infty}\left(0, T ; V_{2}\right)} \leq C \epsilon^{-\frac{1}{2}},
\end{aligned}
$$

where $C$ is a constant independent of $\epsilon$ and $\nu$.

It follows from (1.5) that

$$
\frac{1}{\sqrt{g(s)}} \leq\left|\frac{1}{\sqrt{g(s)}}-\frac{1}{\sqrt{g(0)}}\right|+\frac{1}{\sqrt{g(0)}} \leq C(g)(1+|s|) .
$$

So by (2.23) and (2.15), we have

$$
\|\nabla u\|_{L_{2}\left(0, T ; L_{1}\right)} \leq\|\sqrt{\nu g(v)} \nabla u\|_{L_{2}\left(0, T ; L_{2}\right)}\left\|\sqrt{g(v)}^{-1}\right\|_{L_{\infty}\left(0, T ; L_{2}\right)} \leq C .
$$

By the Sobolev embedding $H_{0}^{1} \subset L_{p}$ for any $p<\infty$ and the Hölder inequality, we derive

$$
\|\nabla u\|_{L_{2}\left(0, T ; L_{1}\right)}+\|\nabla u\|_{L_{1}\left(0, T ; L_{r}\right)}+\|\nabla u\|_{L_{\frac{4}{3}}\left(0, T ; L_{-\epsilon+\frac{4}{3}}\right)} \leq C .
$$

Furthermore, by (2.13)-(2.14) and (2.15), for $1<r<2$ and $0<\epsilon<\frac{1}{3}$, we have the following estimates:

$$
\begin{aligned}
& \|\nabla u\|_{L_{2}\left(0, T ; V_{2}^{*}\right)}+\|v\|_{L_{2}\left(0, T ; H^{-2}\right)} \leq C(1+\sqrt{\epsilon}), \\
& \|v\|_{H^{1}\left(0, T ; H^{-1}\right)} \leq C\left(1+\frac{1}{\sqrt{\epsilon}}\right)
\end{aligned}
$$

where $C$ is a constant independent of $\epsilon$ and $\nu$.

Hence the above estimates imply that

$$
\|u\|_{W_{1}}+\|v\|_{W_{2}}+\left\|v^{\prime}\right\|_{W_{2}} \leq C
$$

where $C$ depends on $\epsilon$ but not on $\nu$.

Applying Schaeffer's theorem (see [23], p.539), we know that there exists a fixed point of (2.8),which is a solution of (2.7). This completes the proof.

The following convergence proposition is taken from [14].

Proposition 2.5 Let $G$ be a measurable set in a finite-dimensional space, $y_{n}: G \longrightarrow R$ be a sequence of functions and $\mathcal{X}: R \rightarrow R$ be a continuous function. Assume that $\left\{y_{n}\right\}$ is uniformly bounded in $L_{\infty}(G)$ and $y_{m} \rightarrow y_{0}$ in $L_{q}(G)$ with $q \geq 1$. Then

$$
\mathcal{X}\left(y_{n}\right) \longrightarrow \mathcal{X}\left(y_{0}\right)
$$

in $L_{p}(G)$ for any $p<\infty$. 
Now we are ready to prove Theorem 2.3. The proof is similar to that of Theorem 1 in [14]. For completeness of our paper, we sketch the proof. Based on Lemma 2.4, we can proceed with the sketch of the proof of Theorem 2.3. We refer to [14] for the details of the technique, and we mainly focus on the new issues. The existence of dissipative solutions, one passes the limit in (2.9) with $v=1$ as $\epsilon=\epsilon_{m} \rightarrow 0$ on every interval $(0, T)$ with $T>0$. Let $\left(u_{n}, v_{n}\right)$ be the weak solution to problem (2.5)-(2.6) with $\epsilon_{n}$ in Lemma 2.4. Using the Sobolev embedding $W_{1} \subset L_{2}$, we derive

$$
\begin{aligned}
& u_{m} \longrightarrow u \quad \text { in } L_{\frac{4}{3}}\left(0, T ; L_{2}\right), \\
& v_{m} \longrightarrow v \quad \text { in } H^{1}\left(0, T ; L_{2}\right) .
\end{aligned}
$$

Then by (2.21) and Proposition 2.5,

$$
\begin{aligned}
& \gamma^{\left\|u_{n}(t)\right\|^{2}} \longrightarrow \gamma^{\|u(t)\|^{2}} \quad \text { in } L_{2}(0, T), \\
& \left\|u_{n}(t)-\psi(t)\right\|^{2} \longrightarrow\|u(t)-\psi(t)\|^{2} \quad \text { in } L_{2}(0, T), \\
& \left\|v_{n}(t)-\phi(t)\right\|^{2} \longrightarrow\|v(t)-\phi(t)\|^{2} \quad \text { in } L_{2}(0, T), \\
& \left\|v_{n}^{\prime}(t)-\phi^{\prime}(t)\right\|^{2} \longrightarrow\|v(t)-\phi(t)\|^{2} \quad \text { in } L_{2}(0, T) \text {. }
\end{aligned}
$$

So we have

$$
\begin{gathered}
\gamma^{\left\|u_{n}(t)\right\|^{2}}\left(\left\|u_{n}(t)-\psi(t)\right\|^{2}+\left\|v_{n}(t)-\phi(t)\right\|^{2}+\left\|v_{n}^{\prime}(t)-\phi^{\prime}(t)\right\|^{2}\right) \\
\longrightarrow \gamma^{\|u(t)\|^{2}}\left(\|u(t)-\psi(t)\|^{2}+\|v(t)-\phi(t)\|^{2}+\left\|v^{\prime}(t)-\phi^{\prime}(t)\right\|^{2}\right)
\end{gathered}
$$

in $L_{1}(0, T)$. Thus, we can pass to the limit in the right-hand side of (2.9) as well and the last summand (the one with $\epsilon$ ) goes to zero. Therefore, we conclude that Theorem 2.3 holds.

\section{Numerical experiments}

In this section, using Rothe's method in time discretization and finite difference method in spatial discretization, we show some experimental results on pictures in the twodimensions case. Let $N$ be a positive integer. The lattice is denoted by $\{1 h, 2 h, \ldots, M h\} \times$ $\{1 h, 2 h, \ldots, L h\}$, where $h$ is the space stepsize. In the discrete numerical algorithm, we subdivide the time interval $(0, T)$ by points $t_{n}=n \tau$ with $\tau=\frac{T}{L}, n=0,1,2, \ldots, N$. Assume that the image $(u(t), v(t))$ is defined in the lattice. Denote by $\left(u_{i, j}^{n}, v_{i, j}^{n}\right)$ an approximation of $(u(n \tau, i h, j h), v(n \tau, i h, j h))$. Define the discrete approximation

$$
\begin{array}{ll}
\nabla_{x}^{+} u_{i, j}^{n}=\frac{u_{i+1, j}^{n}-u_{i, j}^{n}}{h}, & \nabla_{x}^{+} v_{i, j}^{n}=\frac{v_{i+1, j}^{n}-v_{i, j}^{n}}{h}, \\
\nabla_{x}^{-} u_{i, j}^{n}=\frac{u_{i-1, j}^{n}-u_{i, j}^{n}}{h}, & \nabla_{x}^{-} v_{i, j}^{n}=\frac{v_{i-1, j}^{n}-v_{i, j}^{n}}{h}, \\
\nabla_{y}^{+} u_{i, j}^{n}=\frac{u_{i, j+1}^{n}-u_{i, j}^{n}}{h}, & \nabla_{y}^{+} v_{i, j}^{n}=\frac{v_{i, j+1}^{n}-v_{i, j}^{n}}{h}, \\
\nabla_{y}^{-} u_{i, j}^{n}=\frac{u_{i, j-1}^{n}-u_{i, j}^{n}}{h}, & \nabla_{y}^{-} v_{i, j}^{n}=\frac{v_{i, j-1}^{n}-v_{i, j}^{n}}{h},
\end{array}
$$




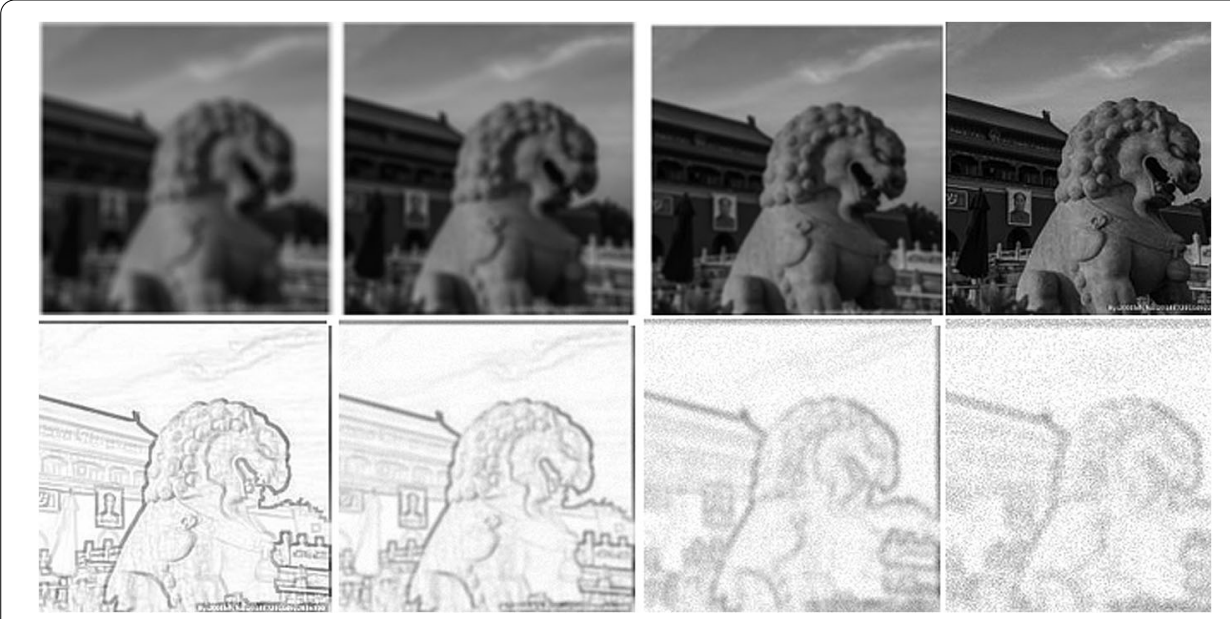

Figure 1 Artificial heavily noised image. (pic 1) Original noise-free image. (pic 2) Heavily noised image with $\mathrm{SNR}=1.24$. (pic 3) Image restored using the present model with $\tau=0.21, \lambda=21, K=7, \sigma=1.5$, and 662 iterations, SNR $=10.40$. (pic 4) Image restored using the (1.1)-(1.2), (1.2) with $\tau=0.3, \lambda=21, K=7$, and 5,151 iterations, $\mathrm{SNR}=9.06$.

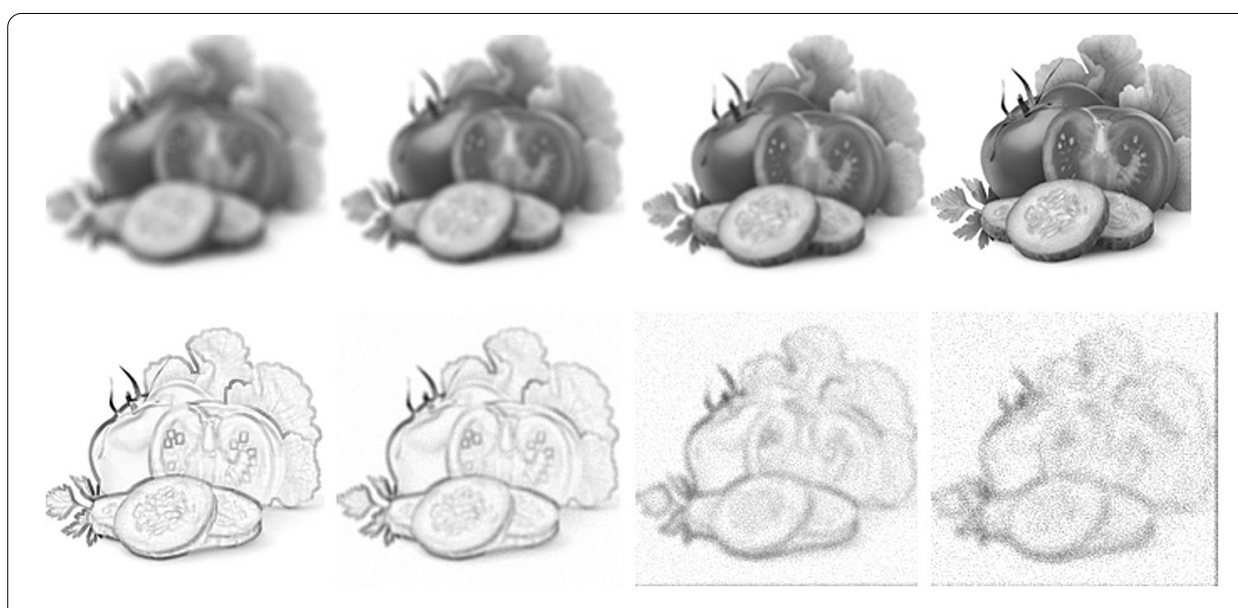

Figure 2 Artificial heavily noised image. (pic 1) Original noise-free image. (pic 2) Heavily noised image with $\mathrm{SNR}=2.25$. (pic 3) Image restored by the present model with $\tau=0.21, \lambda=21, K=7, \sigma=1.2$, and 662 iterations, SNR $=10.30$. (pic 4) Image restored by (1.1)-(1.2), (1.2) with $\tau=0.3, \lambda=21, K=7$, and 5,151 iterations, $\mathrm{SNR}=9.06$.

$$
\begin{array}{ll}
\delta u_{i, j}^{n}=\frac{u_{i, j}^{n}-u_{i, j}^{n-1}}{\tau}, & \delta v_{i, j}^{n}=\frac{v_{i, j}^{n}-v_{i, j}^{n-1}}{\tau}, \\
\delta^{2} v_{i, j}^{n}=\frac{\delta v_{i, j}^{n}-\delta v_{i, j}^{n-1}}{\tau}, & g_{i, j}^{n}=\frac{1}{1+\left(\frac{v_{i, j}^{n}}{K}\right)^{2}} .
\end{array}
$$

Then the discrete explicit scheme of the problem (1.1)-(1.2) can be obtained:

$$
\begin{aligned}
& \delta u_{i, j}^{n+1}-\frac{1}{2}\left[\left(g_{i+1, j}^{n}+g_{i, j}^{n}\right) \nabla_{x}^{+} u_{i, j}^{n}+\left(g_{i-1, j}^{n}+g_{i, j}^{n}\right) \nabla_{x}^{-} u_{i, j}^{n}\right. \\
& \left.\quad+\left(g_{i, j+1}^{n}+g_{i, j}^{n}\right) \nabla_{y}^{+} u_{i, j}^{n}+\left(g_{i, j-1}^{n}+g_{i, j}^{n}\right) \nabla_{y}^{-} u_{i, j}^{n}\right]=0, \\
& \delta^{2} u_{i, j}^{n+1}+\delta u_{i, j}^{n+1}-\frac{1}{2}\left[\left(c_{i+1, j}^{n}+c_{i, j}^{n}\right) \nabla_{x}^{+} v_{i, j}^{n}+\left(c_{i-1, j}^{n}+c_{i, j}^{n}\right) \nabla_{x}^{-} v_{i, j}^{n}\right.
\end{aligned}
$$




$$
\begin{aligned}
& \left.+\left(c_{i, j+1}^{n}+c_{i, j}^{n}\right) \nabla_{y}^{+} v_{i, j}^{n}+\left(c_{i, j-1}^{n}+c_{i, j}^{n}\right) \nabla_{y}^{-} v_{i, j}^{n}\right] \\
& -(1-\lambda)\left(\left|\nabla_{x}^{+} u_{i, j}^{n}\right|+\left|\nabla_{x}^{-} u_{i, j}^{n}\right|-v_{i, j}^{n}\right)=0,
\end{aligned}
$$

with the conditions

$$
\begin{array}{lll}
u_{i, j}^{0}=u_{0}(i, j), & v_{i, j}^{0}=v_{0}(i, j), & \delta v_{i, j}^{0}=\delta v_{0}(i, j), \quad 1 \leq i \leq M, 1 \leq j \leq L, \\
u_{0, j}^{n}=u_{1, j}^{n}, & u_{M, j}^{n}=u_{M+1, j}^{n}, & u_{i, 0}^{n}=u_{i, 1}^{n}, \quad u_{i, L}^{n}=u_{i, L+1}^{n}, \\
v_{0, j}^{n}=v_{1, j}^{n}, & v_{M, j}^{n}=v_{M+1, j}^{n}, & v_{i, 0}^{n}=v_{i, 1}^{n}, \quad v_{i, L}^{n}=v_{i, L+1}^{n} .
\end{array}
$$

We show numerical results which are obtained by applying the above scheme to two artificial heavily noised images. The image, rescaled for the theoretical results obtained in the previous section, can be applied. Meanwhile, it can stabilize the numerical scheme. Our experiments depend on two parameters: the 'scale' of the diffusion $\lambda$ and the threshold $K$. Define the signal-noise ratio (SNPR) as

$$
\mathrm{SNR}=12 \log _{12}\left(\frac{\sum_{\Omega}\left(u_{i, j}-\bar{u}\right)^{2}}{\sum_{\Omega}\left(n_{i, j}\right)^{2}}\right)
$$

where $\bar{u}$ is the mean of the signal $u_{i, j}, n_{i, j}$ is the noise. The better quality image should have a higher SNR. One can see in the first set of images (Figure 1) that our method could improve the general hyperbolic or parabolic method. Figure 1 illustrates the performance of the proposed approach in real image. Figure 2 tests the denoising effect of our method on a standard digital image. The numerical tests show that the proposed method yields better results in image restoration in the case of real images, which is also useful in the case of artificial heavily noised images.

\section{Competing interests}

The authors declare that they have no competing interests.

\section{Authors' contributions}

All authors read and approved the final manuscript.

\section{Author details}

'School of Information Engineering, Wuhan University of Technology, Wuhan, 430070, People's Republic of China.

${ }^{2}$ School of Mathematics and Information Science, Henan University of Polytechnic, Jiaozuo, Henan 454003, People's

Republic of China.

\section{Acknowledgements}

We sincerely thank the referees for very careful reading of the manuscript and comments. This work was supported by NSFC of China (U1404103).

Received: 8 August 2016 Accepted: 13 October 2016 Published online: 24 October 2016

\section{References}

1. Alvarez, L, Mazorra, L: Signal and image restoration using shock filters and anisotropic diffusion. SIAM J. Numer. Anal. 31(2), 590-605 (1994)

2. Perona, P, Malik, J: Scale-space and edge detection using anisotropic diffusion. IEEE Trans. Pattern Anal. Mach. Intell. 12(7), 629-639 (1990)

3. Gilboa, G, Sochen, N, Zeevi, YY: Image enhancement and denoising by complex diffusion process. IEEE Trans. Pattern Anal. Mach. Intell. 26(8), 1020-1036 (2004)

4. Chan, TF, Marquina, A, Mulet, P: High-order total variation based image restoration. SIAM J. Sci. Comput. 22(2), 503-516 (2000)

5. Qiang, L, Yao, ZA, Ke, YY: Entropy solutions for a fourth-order nonlinear degenerate problem for noise removal. Nonlinear Anal. TMA 67, 1908-1918 (2007) 
6. Chan, TF, Golub, GH, Mulet, P: A nonlinear primal-dual method for total variation-based image restoration. SIAM J. Sci. Comput. 20(6), 1964-1977 (1999)

7. Rudin, LI, Osher, S, Fatemi, E: Nonlinear total variation based noise removal algorithms. Physica D 60, 259-268 (1992)

8. Vogel, CR, Oman, ME: Iterative methods for total variation denoising. SIAM J. Sci. Comput. 17(1), 227-238 (1996)

9. Catte, V, Lions, PL, Morel, JM, Coll, T: Image selective smoothing and edge detection by nonlinear diffusion. SIAM J. Numer. Anal. 29(1), 182-193 (1992)

10. Ratner, V, Zeevi, YY: Image enhancement using elastic manifolds. In: ICIAP 2007, 14th International Conference on Image Analysis and Processing, pp. 769-774 (2007)

11. Chen, Y, Barcelos, CAZ, Mair, BA: Smoothing and edge detection by time-varying coupled nonlinear diffusion equations. Comput. Vis. Image Underst. 82(2), 85-100 (2001)

12. Chen, Y, Bose, P: On the incorporation of time-delay regularization into curvature-based diffusion. J. Math. Imaging Vis. 14(2), 149-164 (2001)

13. Nitzberg, M, Shiota, T: Nonlinear image filtering with edge and corner enhancement. IEEE Trans. Pattern Anal. Mach. Intell. 14(8), 826-833 (1992)

14. Surya Prasath, VB, Vorotnikov, D: On a system of adaptive coupled PDEs for image restoration. J. Math. Imaging Vis. 48, 35-52 (2014)

15. Ambrosio, L, Tortorelli, VM: Approximation of functionals depending on jumps by elliptic functionals via t-convergence. Commun. Pure Appl. Math. 43(8), 999-1036 (1990)

16. Ceccarelli, M: A finite Markov random field approach to fast edge-preserving image recovery. Image Vis. Comput. 25(6), 792-804 (2007)

17. Charbonnier, $P, B$ Blanc-Feraud, L, Aubert, G, Barlaud, M: Deterministic edge-preserving regularization in computed imaging. IEEE Trans. Image Process. 6(2), 298-311 (1997)

18. Geman, S, Geman, D: Stochastic relaxation, Gibbs distribution and the Bayesian restoration of images. IEEE Trans. Pattern Anal. Mach. Intell. 6(6), $721-741$ (1984)

19. Mumford, D, Shah, J: Optimal approximations by piecewise smooth functions and associated variational problems. Commun. Pure Appl. Math. 42(5), 577-685 (1989)

20. Szeliski, R, Zabih, R, Scharstein, D, Veksler, O, Kolmogorov, V, Agarwala, A, Tapen, M, Rother, C: A comparative study of energy minimization methods for Markov random fields with smoothness based priors. IEEE Trans. Pattern Anal. Mach. Intell. 30(6), 1068-1080 (2008)

21. Simon, J: Compact sets in the space $L^{P}(0, T ; B)$. Ann. Mat. Pura Appl. 146, 65-96 (1987)

22. Krasnosel'skii, MA: Topological Methods in the Theory of Nonlinear Integral Equations. Pergamon, Elmsford (1964). Translated by AH Armstrong; translation edited by J Burlak

23. Evans, LC: Partial Differential Equations, 2nd edn. Graduate Studies in Mathematics, vol. 19. Am. Math. Soc., Providence (2010)

\section{Submit your manuscript to a SpringerOpen ${ }^{\ominus}$ journal and benefit from:}

- Convenient online submission

Rigorous peer review

- Immediate publication on acceptance

- Open access: articles freely available online

- High visibility within the field

- Retaining the copyright to your article 